\title{
A pore scale study on fluid flow through two dimensional dual scale porous media with small number of intraparticle pores
}

\author{
Safa Sabet ${ }^{1}$, Moghtada Mobedi ${ }^{1,2 *}$, Turkuler Ozgumus ${ }^{1,3}$ \\ ${ }^{1}$ Izmir Institute of Technology, Mechanical Engineering Department, Urla 35430 Izmir, Turkey \\ ${ }^{2}$ Current address: Faculty of Engineering, 3-5-1 Johoku Naka-ku, Hamamatsu, 432-8561 Japan \\ ${ }^{3}$ Current address: Vocational School, Izmir University of Economics, Balcova 35330 Izmir, Turkey \\ "Corresponding author: e-mail: moghtada.mobedi@shizuoka.ac.jp
}

\begin{abstract}
In the present study, the fluid flow in a periodic, non-isotropic dual scale porous media consisting of permeable square rods in inline arrangement is analyzed to determine permeability, numerically. The continuity and NavierStokes equations are solved to obtain the velocity and pressure distributions in the unit structures of the dual scale porous media for flows within Darcy region. Based on the obtained results, the intrinsic inter and intraparticle permeabilities and the bulk permeability tensor of the dual scale porous media are obtained for different values of inter and intraparticle porosities. The study is performed for interparticle porosities between 0.4 and 0.75 and for intraparticle porosities from 0.2 to 0.8. A correlation based on Kozeny-Carman relationship in terms of inter and intraparticle porosities and permeabilities is proposed to determine the bulk permeability tensor of the dual scale porous media.
\end{abstract}

Keywords: pore level numerical analysis, dual scale porous media, interparticle permeability, intraparticle permeability, bulk permeability.

\section{INTRODUCTION}

The macroscopic approach is a simple and practical method for determination of velocity and pressure fields in a porous medium. However, this method requires the macroscopic transport properties of the porous medium such as permeability and/or Forchheimer coefficient for flows with high inertia effect ${ }^{1,2}$. The permeability is a tensor quantity and it depends on the geometrical parameters of the porous media. It can be obtained numerically and/or experimentally. Recently, improvements in computer and software technologies facilitate the prediction of permeability of a porous medium by using pore scale computational approach. Porous media with simple geometrical parameters can be easily modelled in a computer while methods such as tomography techniques can be employed to obtain digital representation of a heterogeneous porous medium with complex pore shapes. The study of Nakayama et al. ${ }^{3}$ who obtained the permeability for a two dimensional periodic isotropic porous media consisting of square rods, Ozgumus et al. ${ }^{4}$ who studied the effect of pore to throat size ratio on permeability and Kozeny constant, are two samples for determination of permeability by using pore level computational approach.

A dual scale porous medium is a porous structure in which the solid region is also permeable since it involves pores in which fluid can flow. A schematic view of a dual scale porous media is shown in Figure 1. As can be seen, there are two kinds of pores in a dual scale porous medium. The first type of pores which can be called as interparticle pores is the main pores of the porous structure and they exist between the particles. The second type of pores called as intraparticle pores are within the particles since the particles are permeableFurthermore, three types of porosities can be defined for a dual scale porous media as interparticle, intraparticle and bulk porosities. These porosities can be found by using below equalities:

$\varepsilon_{p}=\frac{V_{v p}}{V_{p}} ; \varepsilon_{f}=\frac{V_{v f}}{V_{t}} ; \varepsilon_{t}=\frac{V_{v t}}{V_{t}}$ where $\varepsilon_{p}, \varepsilon_{f}$ and $\varepsilon_{t}$ are the intraparticle, interparticle and bulk porosities, respectively. The volumes of intraparticle, interparticle and total pores are shown by $V_{v p}, V_{v f}$ and $V_{t}$, respectively. Furthermore, $V_{p}$ and $V_{t}$ are the total volumes of solid phase and dual scale porous media. The size of interparticle pores is generally greater than the intraparticle pores and consequently the interparticle porosity is generally greater than the intraparticle porosity. The inter and intraparticle pores are also called as macro and micro pores by some researchers ${ }^{5}$. The fluid flow in a dual scale porous medium can also be classified into two types as inter- and intraparticle flows. Hence three related permeabilities can also be defined for a dual scale porous medium as interparticle, intraparticle and bulk permeabilities relating to flow in particle, between particle and the entire dual scale porous medium.

The application of dual scale porous media is widely faced in daily life, nature and industry. The flowing of a fluid through a fiber mat, woven fiber bundles, multifilament textile fibers, oil filters and fractured porous media are some examples for the application of the fluid flow through a dual scale porous media. Table 1 summarizes some pore level computational studies performed on fluid flow thorough a dual scale porous media. In the first column, the name of researcher and related reference number are given. In the second column, the name of governing equations solved to find velocity and pressure

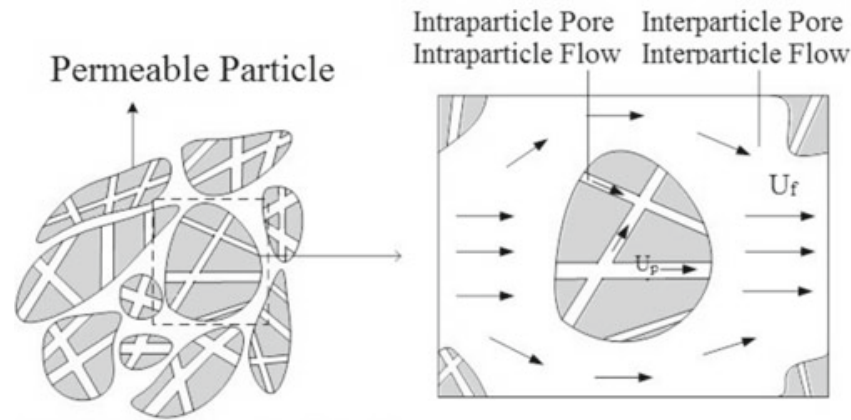

Dual Scale Porous Media 
distributions for inter and intraparticle pores are written. The suggested correlation for the bulk permeability is given in column 3. Finally, the schematic figure of considered dual scale porous media is represented in the last column. As can be seen from Table 1, most of performed studies relates to the porous media with permeable particles containing large number of intraparticle pores. That's why some researchers preferred to solve the Darcy or Brinkman equation to find velocity and pressure distribution for the permeable particle. Some researchers used Stokes or Navier-Stokes equation to find velocity and pressure fields in the permeable particles. An overview on the Table 1 shows that Papathanasiou, Hwang and Advani ${ }^{7}$, Ranganathan ${ }^{8}$, Byon and Kim$^{9}$, $\mathrm{Yu}$ and Cheng ${ }^{5}$, Nield and Kuznetsov ${ }^{10}$ suggested correlations for determination of the bulk permeabilities by using experimental or theoretical methods. Papathanasiou $^{6}$ performed a computational analysis of viscous flow across unidirectional arrays of fiber bundles using the boundary element method. He found a correlation to predict the permeability of fiber bundles in terms of inter and intra-tow porosities (i.e. inter and intra particle porosities), the type of intra-tow packing and the size (or number) of the intra-tow filaments. A study for the full 3-D geometry of an idealized multifilament woven fabric in where the filaments were packed in hexagonal arrangement was done by Hwang and Advani $^{7}$ to compute its permeability and compare with the homogeneous anisotropic lumped model. Ranganathan ${ }^{8}$ developed a predictive semi-analytical solution for flow across arrays of aligned cylinders with elliptical cross sections modeling the fiber mats. The predicted results of the permeability from the model were compared with numerical results obtained from finite element calculations over a range of volume fractions, cross-sectional shapes, and tow permeabilities. Byon and $\mathrm{Kim}^{\mathbf{9}}$ studied the permeability of dual scale porous media fabricated from the monodispersed porous medium which grinded, sieved into specific sizes and sintered into clusters. The inter and intraparticle sizes were 650 and $117 \mu \mathrm{m}$ in their studies. A term involving mean particle diameter and mean cluster size was multiplied to Blake-Kozeny equation to satisfy the obtained numerical and experimental results. $\mathrm{Yu}$ and $\mathrm{Cheng}^{5}$ developed fractal permeability model for dual scale (bi-dispersed) porous media. Their model was based on fractal characteristics of pores in media and it was found that the model should be a function of the tortuosity fractal dimension, pore area fractal dimension, sizes of particles and clusters, micro-porosity inside clusters, and the effective porosity of a medium. The relations suggested by Nield and Kuznetsov ${ }^{\mathbf{1 0}}$ contain three permeabilities as interparticle, intraparticle and bulk permeabilities. The interaction between inter- and intraparticle velocities which causes an extra pressure drop through the dual scale porous media is included by an extra term contains a coefficient $(\zeta)$ called as velocity coupling coefficient.

As it was mentioned before, most of studies on dual scale porous media were performed on porous media consist of permeable particles containing large number of intraparticle pores. However, there are applications (such as cracked rocks) in which limited number of intraparticle pores exist. Hence, the application of Darcy or Darcy-Brinkman equation for intraparticle flow is not correct. For those applications, fluid motion in the intraparticle pores should be solved by Stokes or NavierStokes equations. For the permeable particle with limited number of pore, the direction of the intraparticle pores takes important role in determination of permeability. In this study, a two dimensional porous media consists of permeable square rods in inline arrangement is considered. Each particle in the porous media is splitted by two channels symmetrically and fluid can flow throughout these channels. The Navier-Stokes equations are solved for a representative volume of the porous media and the velocity and pressure distributions for inter and intraparticle voids are found. Based on the obtained results, the intrinsic interparticle permeability (without considering intraparticle flow), intrinsic intraparticle permeability (without considering interparticle flow), and bulk permeability are found. Two relations for determination of bulk permeability tensor in terms of intrinsic inter and intraparticle permeabilities are suggested. To the best of our knowledge, the results of the present study are new and motivate researchers to perform further pore level studies on porous media with cracked particles to develop relationships for determination of bulk permeability.

\section{THE CONSIDERED DUAL SCALE POROUS MEDIA}

The geometry of the considered porous media and representative elementary volume (REV) are shown in Figure 2 and Figure 3. A periodical REV with the dimensions of $\mathrm{H} \times \mathrm{H}$ is chosen as the computational domain. The flow in the REV is assumed fully developed and periodical. The permeable square particles are placed with in-line arrangement. The number of symmetrical intraparticle pores is two in longitudinal flow direction for the entire present study. The height of the intraparticle pore is shown by " $\mathrm{d}$ " and changes from $0.2 \mathrm{D}$ to $0.8 \mathrm{D}$, where $\mathrm{D}$ is the size of square particle. The interparticle porosity is changed between 0.4 and 0.75 while the intraparticle porosity varies between 0.2 and 0.8. The fluid flowing through the medium is assumed to be Newtonian and incompressible with constant thermophysical properties. The flow is laminar and in Darcian region $(\operatorname{Re}<1)$. The study is performed for air with density of $1.225 \mathrm{~kg} / \mathrm{m}^{3}$ and viscosity of $17.894 .10^{-6} \mathrm{~kg} / \mathrm{ms}$.

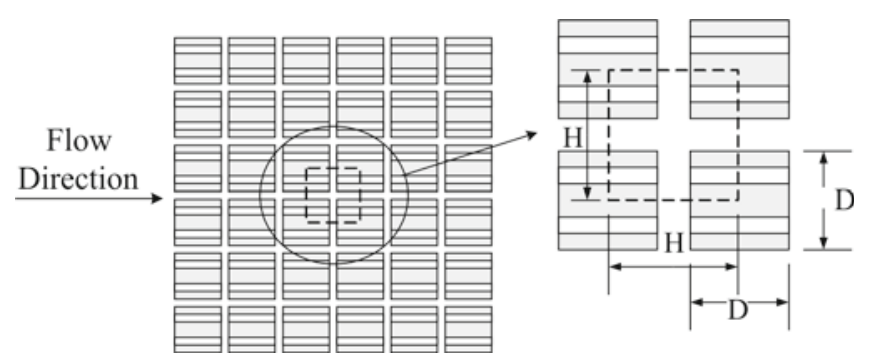

Figure 2. The studied dual scale porous medium

\section{GOVERNING EQUATIONS AND BOUNDARY CONDITIONS}

The fluid flow in inter and intraparticle pores is assumed incompressible and steady. The continuity and momentum equations are solved in order to determine 
Table 1. The performed studies and suggested relations for determination of permeability in a dual scale porous media

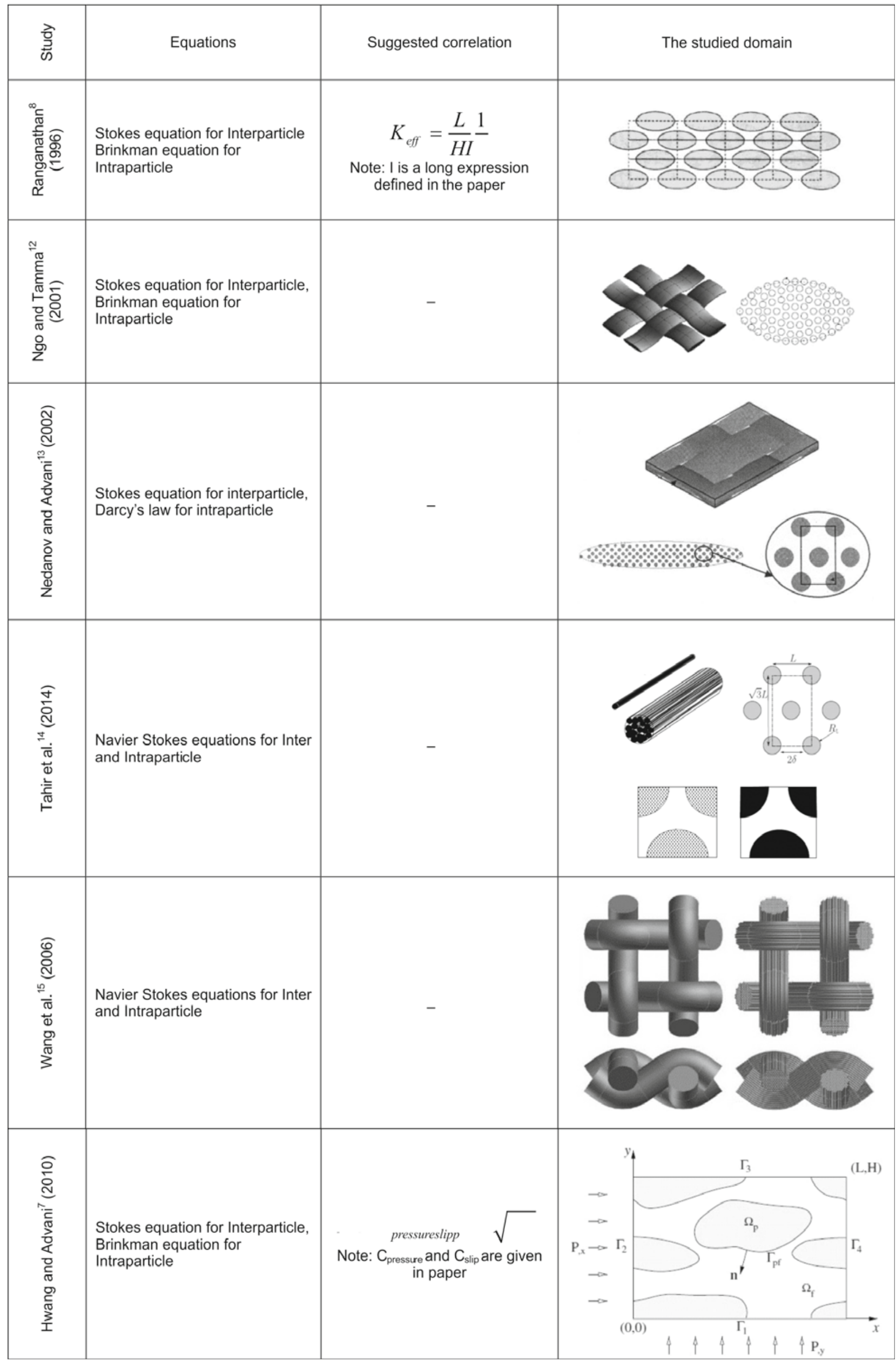


Table 1. The performed studies and suggested relations for determination of permeability in a dual scale porous media (cond. Table 1)

\begin{tabular}{|c|c|c|c|}
\hline 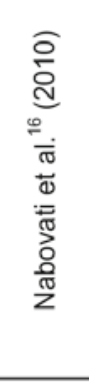 & LBM for Inter and Intraparticle & - & 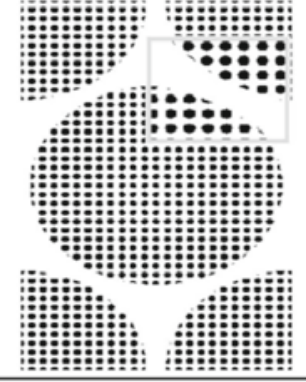 \\
\hline 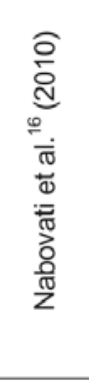 & LBM for Inter and Intraparticle & - & 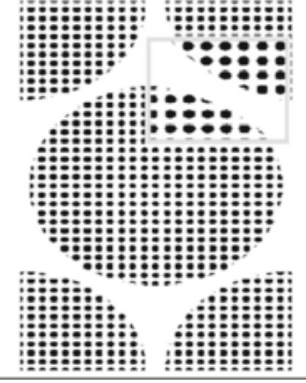 \\
\hline 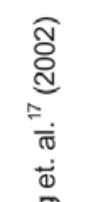 & $\begin{array}{l}\text { Navier Stokes equations for } \\
\text { Interparticle, } \\
\text { Darcy's Law for Intraparticle }\end{array}$ & - & \\
\hline 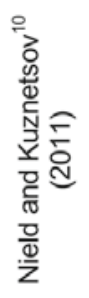 & Theory was developed & $\begin{array}{l}K=\frac{1}{1+(\zeta / \mu)\left(K_{f}+K_{p}\right)} \times \\
\left(\phi K_{f}+(1-\phi) K_{p} 2+(\zeta / \mu) K_{f} K_{p}\right)\end{array}$ & - \\
\hline 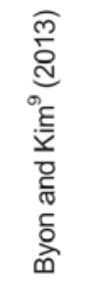 & $\begin{array}{l}\text { Navier Stokes equations for } \\
\text { Interparticle, } \\
\text { Darcy's Law for Intraparticle }\end{array}$ & $\begin{array}{l}K_{b i}=\frac{\varepsilon^{3} D_{c}^{2}}{150(1-\varepsilon)^{2}}[1+ \\
\left.4.03 \exp \left(3.29 \frac{D_{p}}{D_{c}}\right)\left(\frac{D_{p}}{D_{c}}\right)^{2}\right]\end{array}$ & - \\
\hline 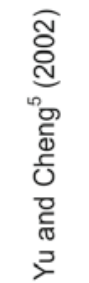 & Fractured model theory & $\begin{array}{l}K=\frac{\pi}{128} \frac{L_{0}^{1-D_{T}}}{A} \times \\
\frac{D_{f}}{3+D_{T}-D_{f}} \lambda_{\max }^{-3+D_{r}}\end{array}$ & \\
\hline
\end{tabular}


a)

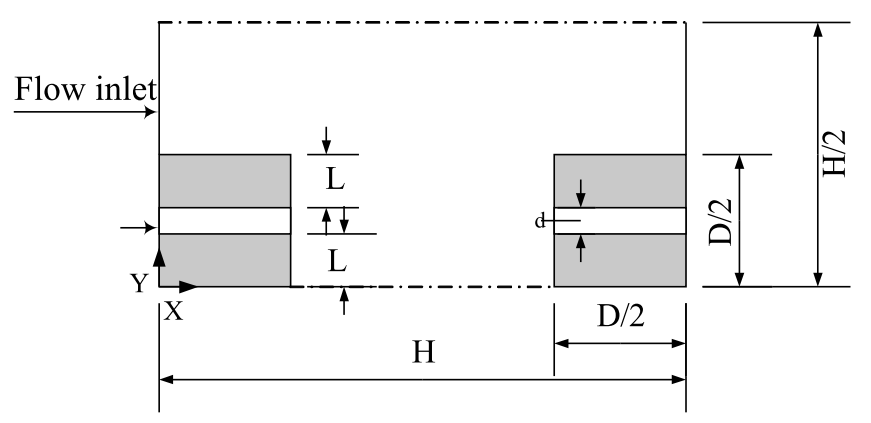

b)

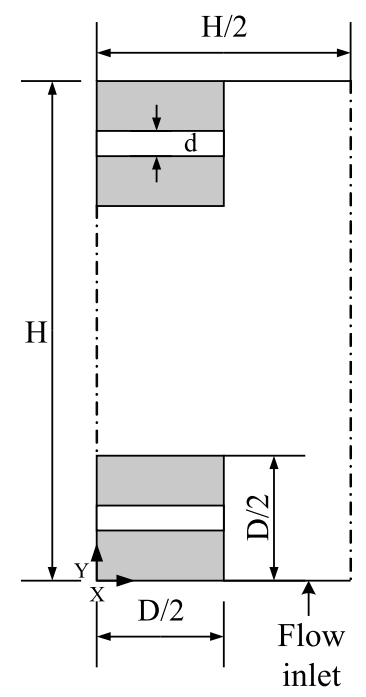

Figure 3. Computational domains a) half of REV for determination of permeability in $\mathrm{x}$ direction $\mathrm{b}$ ) half of REV for determination of permeability in y direction

the velocity and pressure distributions in the pores. These equations in Cartesian coordinate can be written as:

$\frac{\partial u}{\partial x}+\frac{\partial v}{\partial y}=0$

$u \frac{\partial u}{\partial x}+v \frac{\partial u}{\partial y}=-\frac{1}{\rho} \frac{\partial p}{\partial x}+v\left(\frac{\partial^{2} u}{\partial x^{2}}+\frac{\partial^{2} u}{\partial y^{2}}\right)$

$u \frac{\partial v}{\partial x}+v \frac{\partial v}{\partial y}=-\frac{1}{\rho} \frac{\partial p}{\partial y}+v\left(\frac{\partial^{2} v}{\partial x^{2}}+\frac{\partial^{2} v}{\partial y^{2}}\right)$

where $u$ and $v$ are the velocity components in $\mathrm{x}$ and $\mathrm{y}$ directions and $\mathrm{p}$ is the pressure. $\mathrm{Q}$ and $v$ are density and kinematic viscosity of fluid, respectively.

\section{Determination of intrinsic intraparticle permeability}

The intrinsic intraparticle permeability is valid only for $\mathrm{x}$ direction and calculated for a single permeable particle without considering the effect of interparticle flow. The intraparticle permeability is calculated based on velocity field obtained from continuity and momentum equations with the following boundary conditions:

On solid walls: $u=v=0$

For inlet an outlet boundaries:

$u(0, y)=f(y), \frac{\partial u(D / 2, y)}{\partial x}=\frac{\partial v(D / 2, y)}{\partial x}=0$

As it is well known, the permeability depends on the micro-structure of the solid phase in the porous media and it is independent of the properties of the fluid. Generally, the permeability is a tensor quantity and for a two dimensional flow in Cartesian coordinate it can be defined as:

$\left(\begin{array}{l}\langle u\rangle \\ \langle v\rangle\end{array}\right)=\frac{-1}{\mu}\left(\begin{array}{ll}K_{x x} & K_{x y} \\ K_{y x} & K_{y y}\end{array}\right)\left(\begin{array}{c}\frac{\partial\langle p\rangle^{f}}{\partial x} \\ \frac{\partial\langle p\rangle^{f}}{\partial y}\end{array}\right)$

where $K_{x y}, K_{y x}, K_{x x}, K_{y y}$ are components of the permeability tensor.

The Darcy velocity and pressure gradient for flow through the quarter of intraparticle pore is calculated by following relation:

$\left\langle u_{p}\right\rangle=\frac{4}{D^{2}} \int_{L}^{L+d} \int_{0}^{\mathrm{D} / 2} u d x d y$

$-\frac{d\langle p\rangle^{f}}{d x}=\frac{2}{D d}\left[\left.\int_{L}^{L+d} p\right|_{x=0} d y-\left.\int_{L}^{L+d} p\right|_{x=D / 2} d y\right]$

For the intrinsic intraparticle permeability the permeability tensor takes the following form:

$\left(\begin{array}{l}\left\langle u_{p}\right\rangle \\ \left\langle v_{p}\right\rangle\end{array}\right)=\frac{-1}{\mu}\left(\begin{array}{cc}K_{p, x x} & 0 \\ 0 & 0\end{array}\right)\left(\begin{array}{c}\frac{\partial\langle p\rangle^{f}}{\partial x} \\ \frac{\partial\langle p\rangle^{f}}{\partial y}\end{array}\right)$

where $K_{p, x x}$ is the permeability component in longitudinal direction. The value of $K_{p, x y}$ and $K_{p, y x}$ and the value of $K_{p, y y}$ component vanished.

\section{Determination of intrinsic interparticle permeability}

The intrinsic interparticle permeability (without considering of the intraparticle pores) is found by using the continuity and momentum equations with following boundary conditions for flow in $\mathrm{x}$ direction:

On solid walls: $u=v=0$

For inlet and outlet boundaries:

$u(0, y)=f(y), \mathrm{v}(0, \mathrm{y})=0$ and $\frac{\partial u(H, y)}{\partial x}=\frac{\partial v(H, y)}{\partial x}=0$

The Darcy velocity and pressure gradient in $\mathrm{x}$ direction for flow through the half of REV is calculated by following relation:

$\left\langle u_{f}\right\rangle=\frac{2}{H^{2}} \int_{0}^{H / 2} \int_{0}^{H} u d x d y$

$-\frac{d\langle p\rangle^{f}}{d x}=\frac{2}{H(H-D)}\left[\left.\int_{D / 2}^{(H-D) / 2} p\right|_{x=0} d y-\left.\int_{D / 2}^{(H-D) / 2} p\right|_{x=H} d y\right]$

For the intrinsic interparticle permeability, the permeability tensor takes the following form:

$\left(\begin{array}{c}\left\langle u_{f}\right\rangle \\ \left\langle v_{f}\right\rangle\end{array}\right)=\frac{-1}{\mu}\left(\begin{array}{cc}K_{f, x x} & 0 \\ 0 & K_{f, y y}\end{array}\right)\left(\begin{array}{l}\frac{\partial\langle p\rangle^{f}}{\partial x} \\ \frac{\partial\langle p\rangle^{f}}{\partial y}\end{array}\right)$

For the studied case the values of $K_{f, x x}$ and $K_{f, y y}$ are equal to each other due to symmetrical geometry of REV.

\section{Determination of bulk permeability}

The bulk permeability in $\mathrm{x}$ and $\mathrm{y}$ directions are different, that's why they should be calculated for both sides. The bulk permeability is determined by solution of the governing equations and boundary conditions; 
For the determination of the permeability in the horizontal direction:

On solid walls: $u=v=0$

For top and bottom boundaries:

$\frac{\partial u}{\partial y}=\frac{\partial v}{\partial y}=0$

For inlet and outlet boundaries:

$u(0, y)=f(y), \mathrm{v}(0, \mathrm{y})=0$ and $\frac{\partial u(H, y)}{\partial x}=\frac{\partial v(H, y)}{\partial x}=0$

For determination of the permeability in the vertical direction:

On solid walls: $u=v=0$

For left and right boundaries:

$\frac{\partial u}{\partial y}=\frac{\partial v}{\partial y}=0$

For inlet and outlet boundaries:

$v(x, 0)=f(x), u(x, 0)=0$ and $\frac{\partial u(x, H)}{\partial y}=\frac{\partial v(x, H)}{\partial y}=0$

The functions of $\mathrm{f}(\mathrm{x})$ and $\mathrm{f}(\mathrm{y})$ are the velocity profile for the inlet boundary of the REV.

After obtaining periodic velocity field in the structural units, the macroscopic velocities (Darcian velocities) are calculated by using following equations:

$\left\langle u_{b}\right\rangle=\frac{2}{H^{2}} \int_{0}^{H / 2} \int_{0}^{H} u d x d y$

$\left\langle v_{b}\right\rangle=\frac{2}{H^{2}} \int_{0}^{H / 2} \int_{0}^{H} v d y d x$

For determination of permeability in $\mathrm{x}$ and $\mathrm{y}$ direction, the pressure drop throughout half of REV is found by following relations:
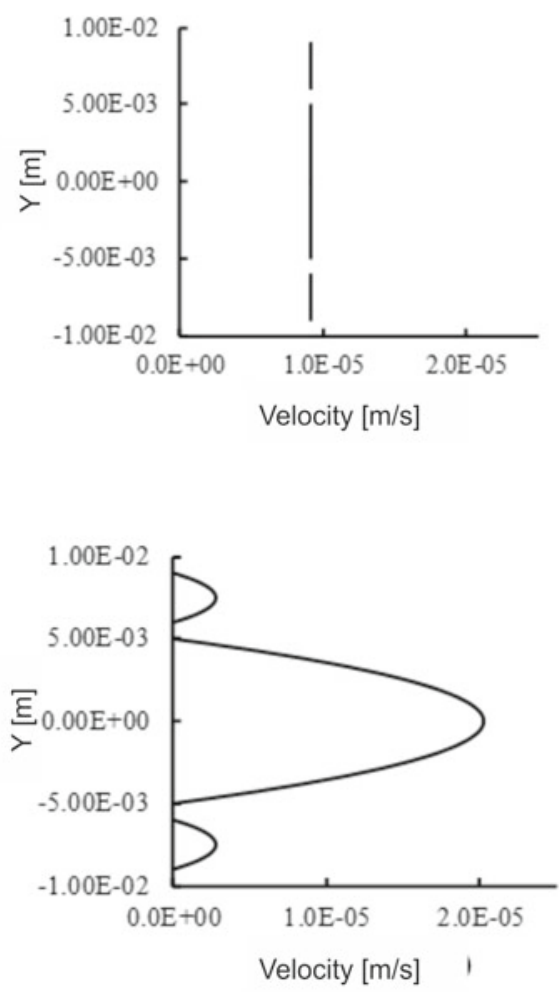

$-\frac{d\langle p\rangle^{f}}{d x}=\frac{1}{H}\left[\frac{\left.\int_{L}^{L+d} p\right|_{x=0} d y+\left.\int_{D / 2}^{(H-D) / 2} p\right|_{x=0} d y}{(H-D) / 2+d}-\frac{\left.\int_{L}^{L+d} p\right|_{x=H} d y+\left.\int_{D / 2}^{(H-D) / 2} p\right|_{x=H} d y}{(H-D) / 2+d}\right]$

$-\frac{d\langle p\rangle^{f}}{d y}=\frac{2}{H(H-D)}\left[\left.\int_{D / 2}^{(H-D) / 2} p\right|_{y=0} d x-\left.\int_{D / 2}^{(H-D) / 2} p\right|_{y=H} d x\right]$

The first term of the right hand side of Eq. (24) is the integral of pressure in inlet while the second term gives the integral of pressure drop in the outlet of REV. As it was mentioned before, permeability is a tensor quantity and for bulk permeability it can be given as follows:

$\left(\begin{array}{l}\left\langle u_{b}\right\rangle \\ \left\langle v_{b}\right\rangle\end{array}\right)=\frac{-1}{\mu}\left(\begin{array}{cc}K_{b, x x} & 0 \\ 0 & K_{b, y y}\end{array}\right)\left(\begin{array}{l}\frac{\partial\langle p\rangle^{f}}{\partial x} \\ \frac{\partial\langle p\rangle^{f}}{\partial y}\end{array}\right)$

Since the structure of REV in $\mathrm{x}$ and $\mathrm{y}$ directions are different, hence $K_{x x} \neq K_{y y}$.

\section{NUMERICAL PROCEDURE}

The pore level flow equations are solved for the studied REVs. The number of grids is chosen as $400 \times 400$ for the entire domain. A commercial code based on finite volume method is used to solve the governing equations, computationally. The power law scheme is employed to treat the discretization of the convection terms in the momentum equation. SIMPLE method is used for handling the pressure-velocity coupling. Figure 4 shows the inlet and outlet velocity profiles obtained for determination of the bulk permeability. Firstly, a uniform velocity profile is assumed as seen from Figure 4(a). After solving the governing equations and obtaining the velocity field, the outlet velocity profile of first run is used as the inlet
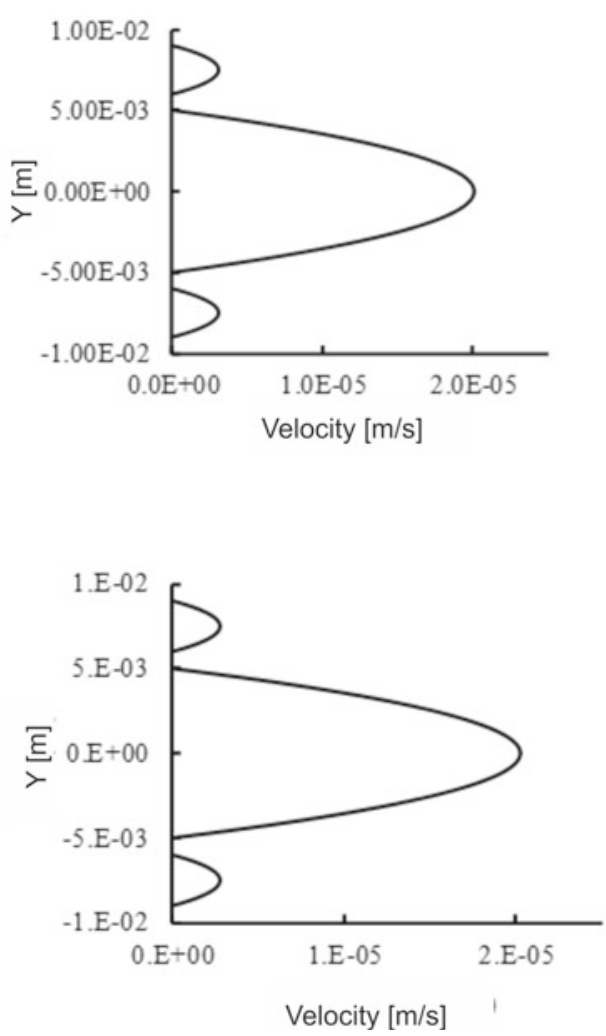

Figure 4. The change of velocity profile to obtain fully developed condition a) inlet for the first run, b) outlet of first run, inlet for the second run, c) outlet of the second run, inlet for the third run, d) outlet of the third run 
boundary condition for second run (Fig. 4 (b)) and the problem is solved again. This iteration continues until the same velocity profiles at the inlet and outlet boundaries achieved (Fig. 4 (c), (d)). By this way, the periodicity of the velocity field is attained. The same method is applied for obtaining flow periodicity in y direction. The approximate errors (Residuals) are set to $10^{-9}$ for flow variables. Grid independency study is done and the results for cases with $\varepsilon_{f}=0.75\left(\varepsilon_{p}=0.2\right)$ and $\varepsilon_{f}=0.4\left(\varepsilon_{p}=\right.$ 0.8 ) when $\mathrm{Re}=0.01$ are displayed in Figure 5. As can be seen, discretization with grid number of 160000 is sufficient to achieve acceptable results.

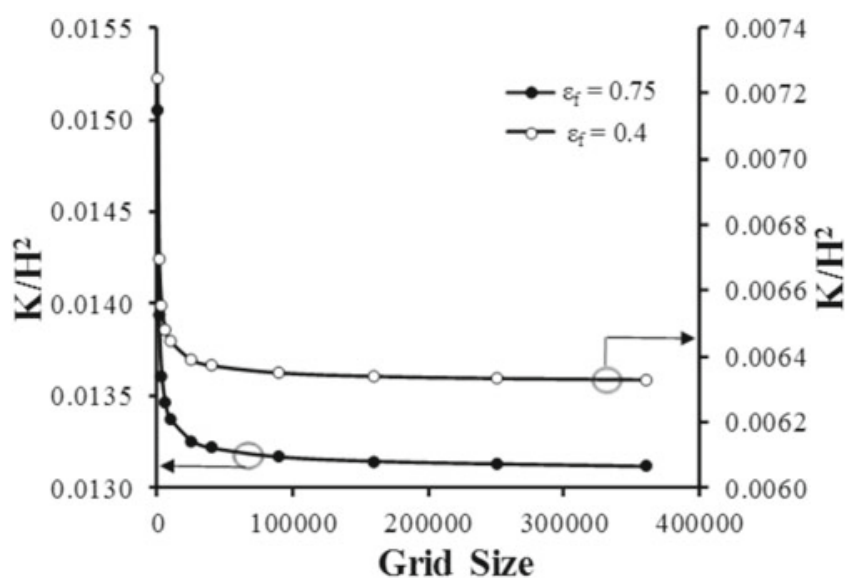

Figure 5. The change of dimensionless permeability with grid number for two dual scale porous media $\varepsilon_{f}=0.75$, $\varepsilon_{p}=0.2$ and $\varepsilon_{f}=0.4, \varepsilon_{p}=0.8$

\section{RESULTS AND DISCUSSION}

Figure 6 shows the change of dimensionless pressure drop with different Reynolds number $(0.01<\operatorname{Re}<1)$ for two porous media as $\varepsilon_{f}=0.75, \varepsilon_{p}=0.2$ and $\varepsilon_{f}=0.4$, $\varepsilon_{p}=0.8$. As well known, for small values of Reynolds number, the permeability does not change with flow and it should remain constant. This figure shows that Darcy equation is valid and inertia effect is negligible for all results obtained in this study. In Figure 7 the obtained dimensionless permeability for porous media with impermeable particle are compared with the values reported by Ozgumus et $\mathrm{al}^{4}$, and Saada et al. ${ }^{11}$ and Na-

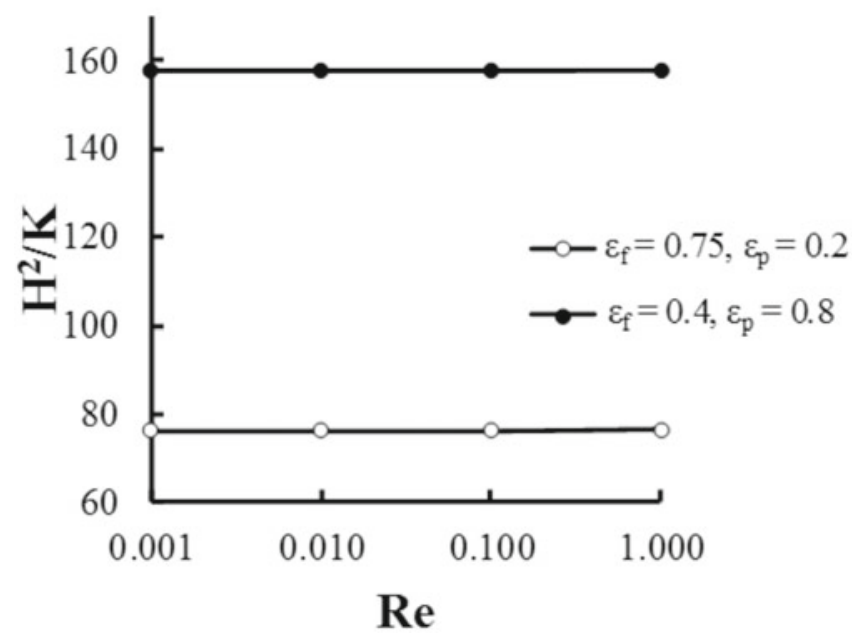

Figure 6. Change of permeability with Reynolds number for two dual scale porous media $\varepsilon_{f}=0.75, \varepsilon_{p}=0.2$ and $\varepsilon_{f}=0.4, \varepsilon_{p}=0.8$

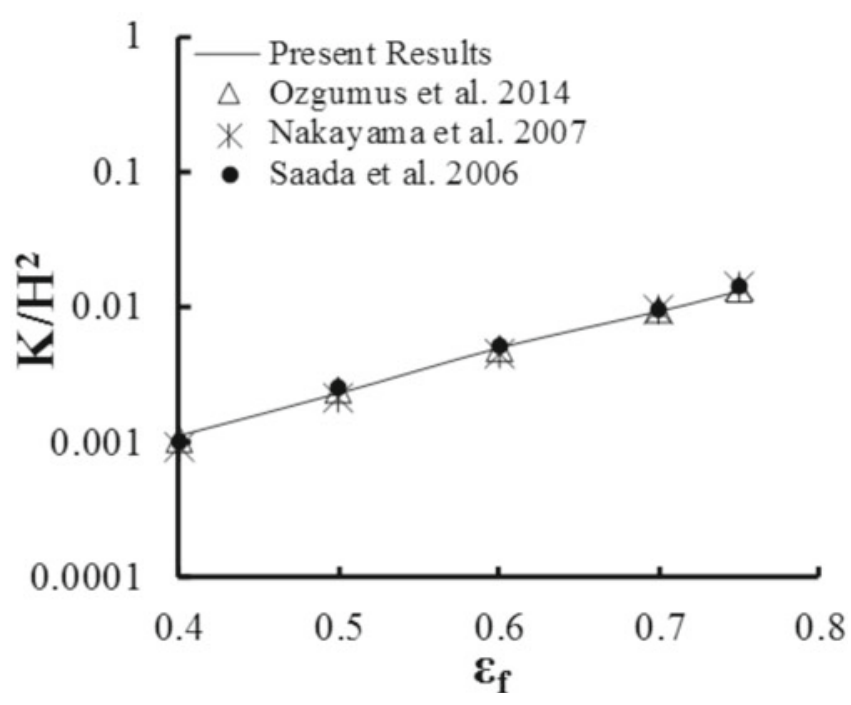

Figure 7. Comparison of permeability obtained in this study with the results reported in literature

kayama et al. $^{\mathbf{3}}$, for square rod porous media when $\mathrm{Re}$ $=0.01$. As seen from the figure, the obtained values of the present study and the reported literature values are in good agreement. The intrinsic and bulk permeabilities both in $\mathrm{x}$ and $\mathrm{y}$ directions are found and presented in this section, separately.

\section{Permeability in $\mathbf{x}$ Direction}

Figure 8 shows the streamlines and normalized pressure distributions in inter and intraparticle pores of dual scale porous media with different intraparticle porosities when $\varepsilon_{f}=0.75$. In this figure, the pressure of each porous media is added with a constant value such that the inlet pressure values of all REVs become identical. Then, the normalization is done by dividing of the pressure in entire REV with the identical inlet pressure value. Figure 8 (a) shows the streamlines and pressure distribution for a dual scale porous medium with $\varepsilon_{p}=0.2$. As seen, inter and intraparticle flows provide the fluid transfer from one REV to another as a result of pressure gradient. The secondary flows occur in the top and bottom gaps between two rods. Figure 8 (b) shows the flow patterns in the REV of the same porous media however the intraparticle porosity is increased to 0.4. The same types of flows are also observed for this porous structure. The flow rate in intraparticle region increases, however, the size of the secondary flows in the top and bottom gaps decreases. In Figure 8 (c) and (d), the intraparticle porosity increases to $\varepsilon_{f}=0.6$ and 0.8 , respectively and the interparticle porosity remains constant. The secondary flows on the top and bottom of the REVs disappear and the flow rate of intraparticle becomes comparable with the rate of interparticle flow. In Figure 9, the streamlines and pressure distributions in the interparticle and intraparticle pores of dual scale porous media with different intraparticle porosities when $\varepsilon_{f}=0.4$ are shown. Figure 9 (a) shows the streamlines and pressure distribution for a dual scale porous medium with $\varepsilon_{p}=0.2$. The interparticle flow is dominant in the horizontal direction. The secondary flows occur in the top and bottom gaps are compressed by the intraparticle flows. The main flow passes through the interparticle region while the rate of flow in the intraparticle pores are smaller. Figure 9 (b) indicates the flow patterns in 

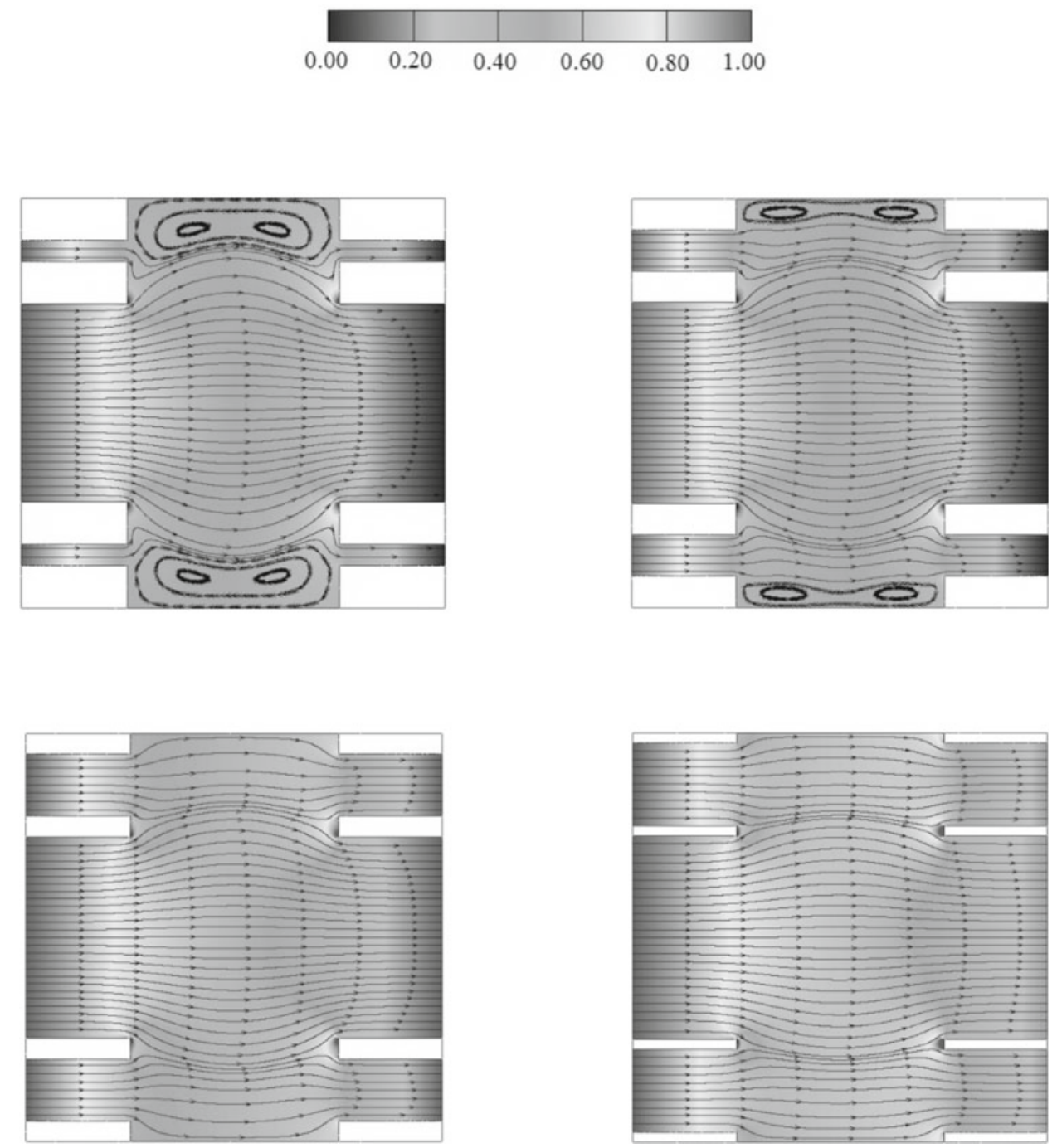

Figure 8. The streamlines and pressure contours for flow in $\mathrm{x}$ direction in dual scale porous media with $\varepsilon_{f}=0.75$, a) $\varepsilon_{p}=0.2$, b) $\varepsilon_{p}=0.4$, c) $\varepsilon_{p}=0.6$, d) $\varepsilon_{p}=0.8$

REV with $\varepsilon_{p}=0.4$ for the same interparticle porosity. The size of the secondary flow decreases and flow rate passes through the intraparticle region increases. In Figure 9 (c) and (d) are plotted for extreme cases for which the intraparticle porosities are 0.6 and 0.8 . It is observed that the fluid flows through inter and intraparticle parts are comparable or even the intraparticle flow rate is greater than interparticle one.

The variations of intrinsic interparticle, intraparticle and bulk permeabilities with intraparticle porosity are illustrated in Figure 10. In Figure 10 (a), the change of permeability values for $\varepsilon_{f}=0.75$ is shown. As seen, the value of intrinsic interparticle permeability is constant while the value of intrinsic intraparticle permeability increases with the increase of $\varepsilon_{p}$. The values of interparticle and bulk permeabilities are higher than the intraparticle permeability even for $\varepsilon_{p}=0.8$. When the interparticle porosity becomes 0.6 (Fig. 10 (b)), the value of intrinsic interparticle permeability becomes lower than the ones of $\varepsilon_{f}=0.75$. The values of intrinsic intraparticle and interparticle permeabilities become closer to each other for $\varepsilon_{p}=0.8$. The change of permeability with $\varepsilon_{p}$ for the interparticle porosity of 0.5 is revealed in Figure 10 (c). The same trend of increasing $K_{p}$ with $\varepsilon_{p}$ observed in Figure 10 (a) and (b), can also be seen. However, for $\varepsilon_{p}$ $=0.5$, the intrinsic inter and intraparticle permeabilities become equal at a point around $\varepsilon_{p}=0.6$. After this value of $\varepsilon_{p}$, the value of $K_{p}$ increases and it becomes greater than interparticle permeability. For $\varepsilon_{p}=0.8$, the values of $K_{p}$ and bulk permeability becomes closer to each other. Figure 10 (d) indicates the permeability variations with $\varepsilon_{p}$ for interparticle porosity of 0.4 . For the intraparticle porosity greater than 0.4 , the effect of intrinsic intraparticle permeability is greater than $K_{f}$ since the fluid flows mainly through intraparticle pores. The domination of intraparticle respect to interparticle permeability for $\varepsilon_{f}=0.4$ and $\varepsilon_{p}=0.8$ can be observed from streamlines in Figure 9 (d).

Figure 11 shows the change of $K_{b, x x} / K_{f, x x}$ with intraparticle porosity for different values of interparticle porosity. As can be seen, for high values of interparticle porosity (such as 0.75) the effect of intra pores is negligible and the value of bulk permeability remains almost constant. Hence, creating intraparticle channels or pores does not influence the bulk permeability. For a porous media with $\varepsilon_{f}=0.75$, creating intraparticle pore with $\varepsilon_{p}=0.8$ can increase the bulk permeability only $23 \%$. For the low values of interparticle porosity the effect of intraparticle pores becomes visible. For a porous media with $\varepsilon_{f}=0.4$, creating pores with the porosity of $\varepsilon_{p}=0.4$ can increase the bulk permeability with $464 \%$.

In Figure 12, the variation of $K_{b, x x} / D^{2}$ with $\varepsilon_{f}^{3} /\left(1-\varepsilon_{f}\right)^{2}$ for different intraparticle porosities is shown. For mono-dispersed porous medium $\left(\varepsilon_{p}=0\right)$ 

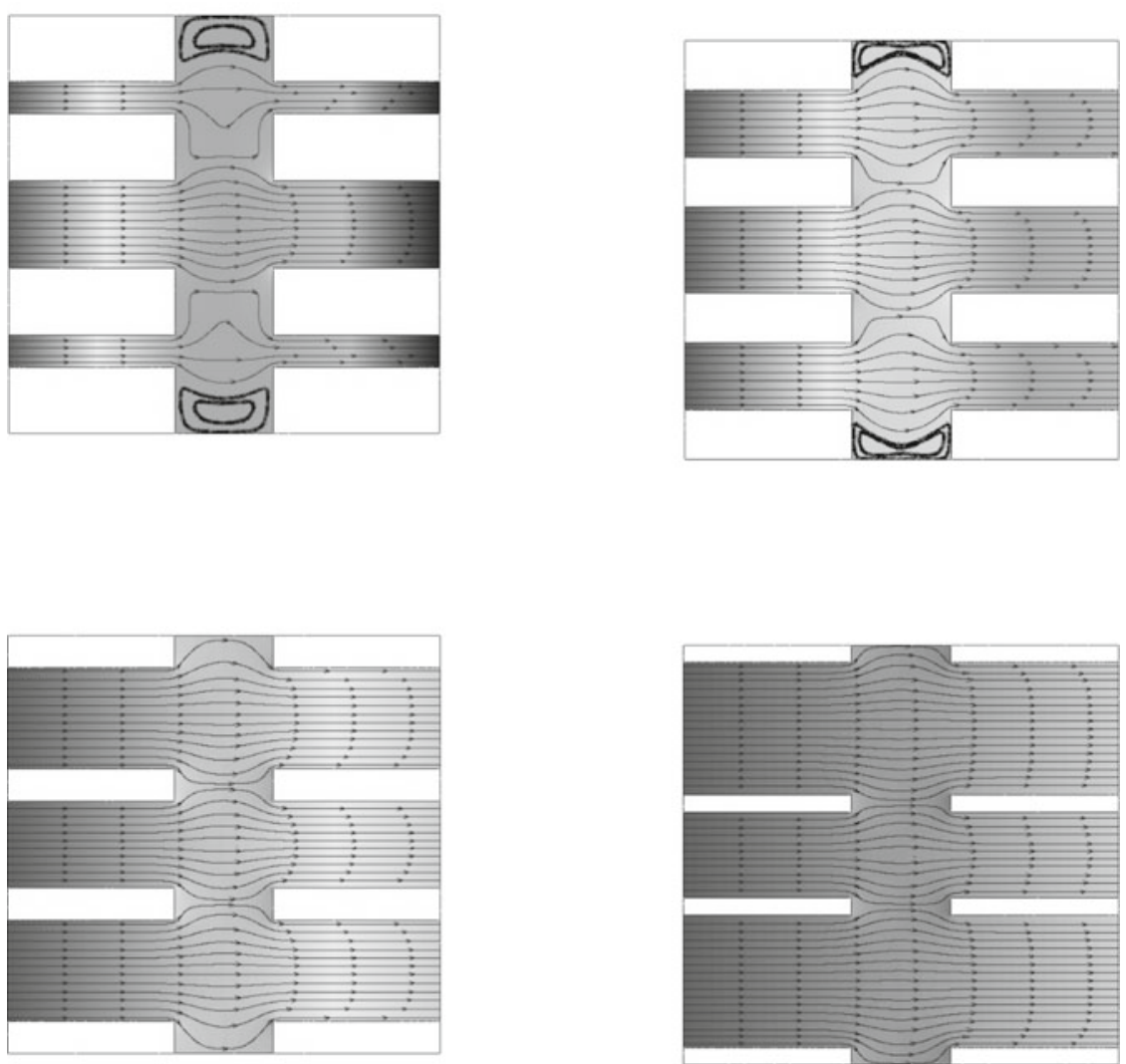

Figure 9. The streamlines and pressure contours for flow in $\mathrm{x}$ direction in dual scale porous media with $\varepsilon_{f}=0.4$, a) $\varepsilon_{p}=0.2$, b) $\left.\left.\varepsilon_{p}=0.4, \mathrm{c}\right) \varepsilon_{p}=0.6, \mathrm{~d}\right) \varepsilon_{p}=0.8$
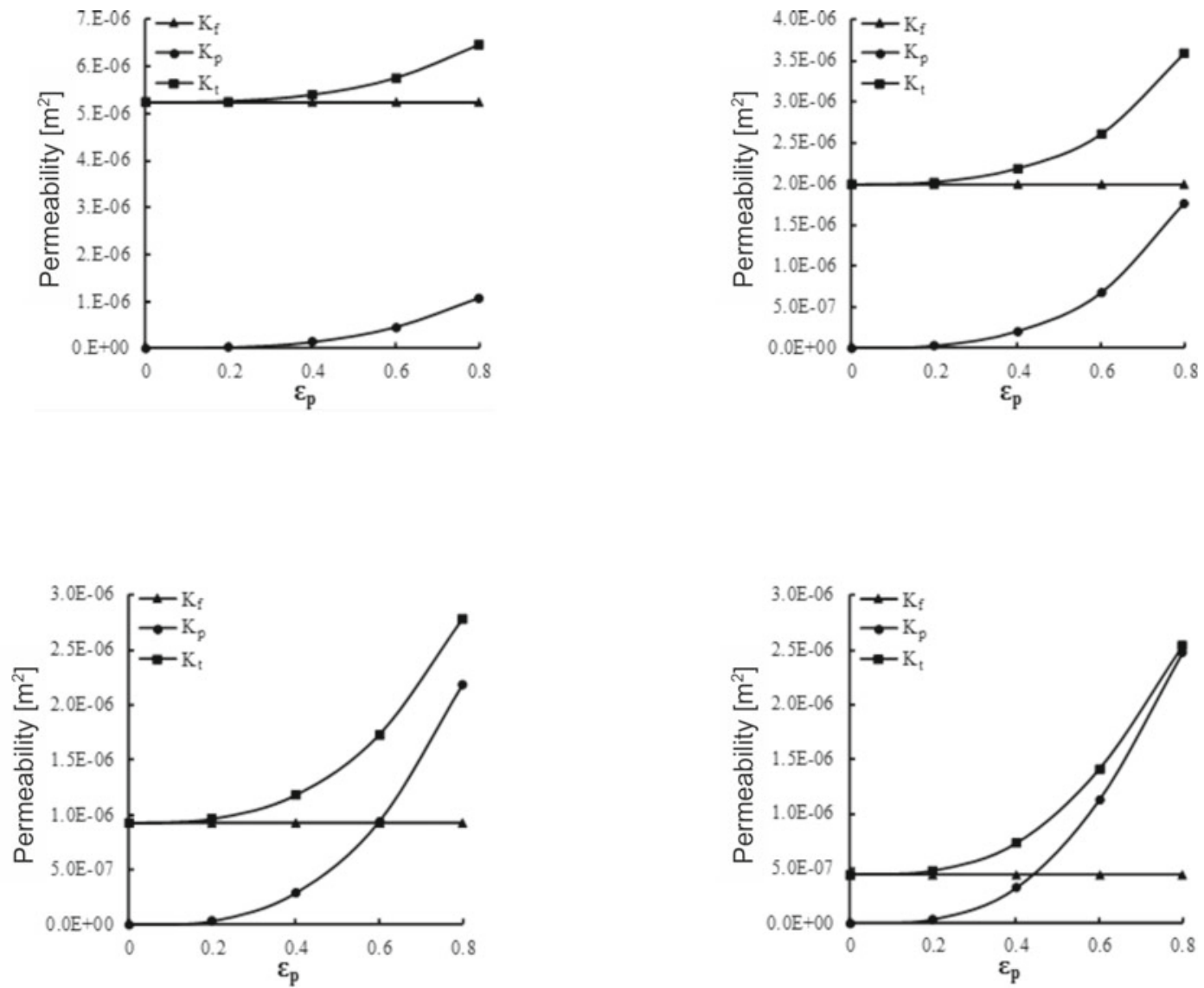

Figure 10. The change of intrinsic interparticle, intraparticle and bulk permeabilities with intraparticle porosity a) $\varepsilon_{f}=0.75$, b) $\varepsilon_{f}=0.6$, c) $\varepsilon_{f}=0.5$, d) $\varepsilon_{f}=0.4$ 


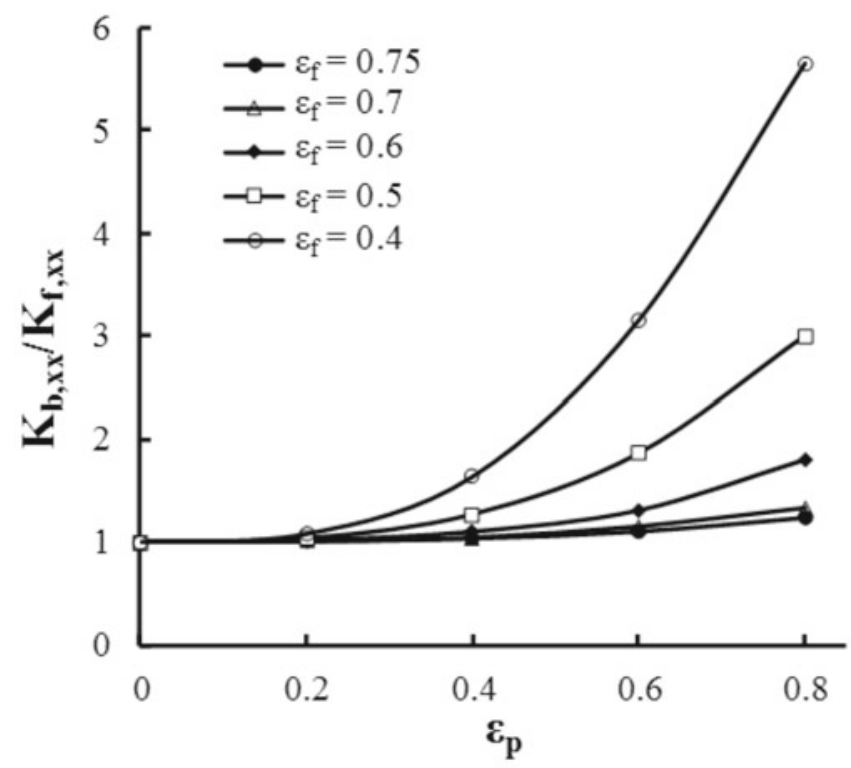

Figure 11. Change of $K_{b, x x} / K_{f, x x}$ with intraparticle porosity for different values of interparticle porosity

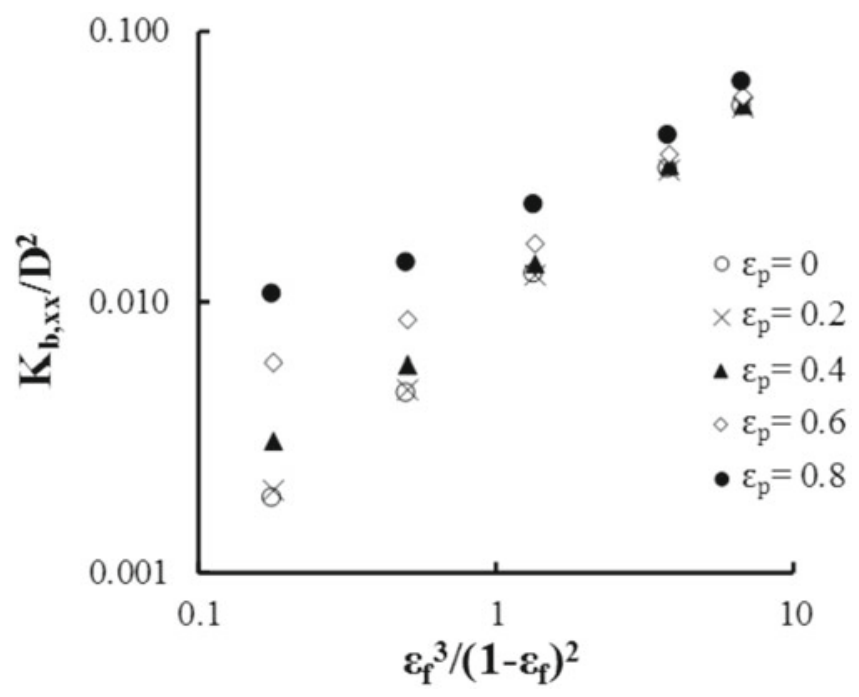

Figure 12. The change of $K_{b, x x} / D^{2}$ with $\varepsilon_{f}^{3} /\left(1-\varepsilon_{f}\right)^{2}$

the change of $K_{b, x x} / D^{2}$ with $\varepsilon_{f}^{3} /\left(1-\varepsilon_{f}\right)^{2}$ seems linear as expected from Kozeny-Carman equation. As the intraparticle porosity increases, the linear relationship between $K_{b, x x} / D^{2}$ and $\varepsilon_{f}^{3} /\left(1-\varepsilon_{f}\right)^{2}$ disappears. As seen, the value of $K_{b, x x}$ increases with $\varepsilon_{f}$, however the rate of increase is different and it is function of $\varepsilon_{p}$. For the large values of $\varepsilon_{f}^{3} /\left(1-\varepsilon_{f}\right)^{2}$, the change of bulk permeability with intraparticle porosity is very small since the size of particles is small in the REV. For small values of $\varepsilon_{f}^{3} /\left(1-\varepsilon_{f}\right)^{2}$, the effect of $\varepsilon_{p}$ is considerable since the size of particles and consequently the volume of intraparticle pores in REV become larger.

\section{Permeability in y Direction}

Figure 13 shows the streamlines and pressure distributions in inter and intraparticle pores of different dual scale porous media when fluid flows only in y direction. In the REVs shown in this figure, the intraparticle porosity is different while the interparticle porosity is constant as $\varepsilon_{f}=0.75$. The main flow (interparticle flow) occurs in the vertical direction and there are secondary flows in the right and left gaps between the particles. The main flow penetrates a little bit into the left and right gaps between the particles and distorts the secondary flows. As seen from the figures, the size of secondary flows in the left and right gaps between the particles is not affected by increasing the value of intraparticle porosity; however the size of vortices in the pores inside the particles changes with intraparticle porosity. One may found that the bulk permeability in y direction is almost identical for all dual scale porous media shown in Figure 13.

Figure 14 shows the streamlines and pressure distribution in inter and intraparticle pores of different dual scale porous media in y direction. In these figures, intraparticle porosity changes while the interparticle porosity remains constant $\varepsilon_{f}=0.4$.Although the increase of intraparticle permeability increases the area of the gap in transverse direction of flow, it seems that the increase of $\varepsilon_{p}$ does not have significant influence on the bulk permeability in y direction. Figure 15 indicates the variation between $K_{b, y y} / D^{2}$ and $\varepsilon_{f}^{3} /\left(1-\varepsilon_{f}\right)^{2}$ for different intraparticle porosities. As seen, the permeability in y direction is not influenced with the change of the intraparticle porosity and there is almost a linear variation between $K_{b, y y} / D^{2}$ and $\varepsilon_{f}^{3} /\left(1-\varepsilon_{f}\right)^{2}$ that enables the use of Kozeny-Carmen equation with constant Kozeny coefficient for determination of $K_{b, y y}$.

\section{Suggested Correlations}

Based on the obtained results, Kozeny constant for $\mathrm{x}$ and $\mathrm{y}$ directions are calculated to find permeability value by using Kozeny-Carman equation. As it is well known, the Kozeny-Carman permeability relation was derived based on a porous medium consist of bundle of capillary channels with same radius. It can be defined as:

$K=\frac{D^{2} \varepsilon_{f}^{3}}{16 \kappa\left(1-\varepsilon_{f}\right)^{2}}$

where $K$ is called as Kozeny constant and it depends on porous media structure. In this study, based on obtained numerical results, Kozeny constant for $\mathrm{x}$ and $\mathrm{y}$ directions are found and presented in following part.

For Kozeny constant in $\mathrm{x}$ direction, a general equation for Kozeny constant based on the ratio of intra and interparticle permeability and intraparticle porosity is obtained. A proper mathematical relationship for the change of Kozeny constant in terms of the permeability ratio for $0.4<\varepsilon_{f}<0.75$ and $0.2<\varepsilon_{p}<0.8$ can be suggested as:

$\kappa=A\left(\frac{K_{p, x x}}{K_{f, x x}}\right)^{B}$

where A and B coefficients are constants and they are functions of intraparticle porosity and can be calculated from the following equation:

$A=C_{0} \varepsilon_{p}{ }^{3}+C_{1} \varepsilon_{p}{ }^{2}+C_{2} \varepsilon_{p}+C_{3}$

$B=D_{0} \varepsilon_{p}{ }^{3}+D_{1} \varepsilon_{p}^{2}+D_{2} \varepsilon_{p}+D_{3}$

Based on the obtained pore level permeability values, the constant coefficients of equations (29) and (30) are obtained and given in Table 2. Figure 16 shows the comparison of the permeability values found by using the suggested Kozeny-Carman relation with the val- 

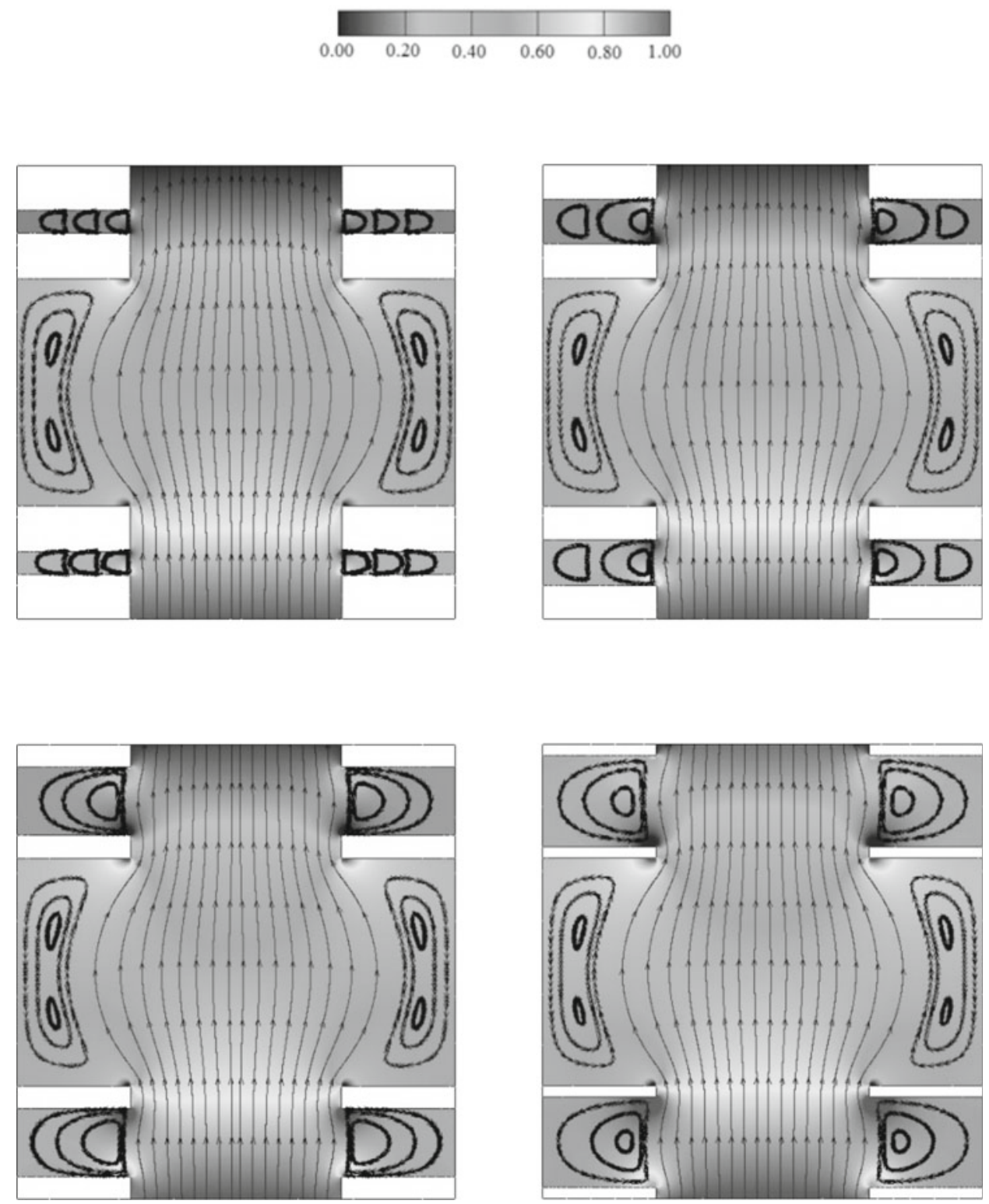

Figure 13. The streamlines and pressure contours for flow in $\mathrm{y}$ direction in dual scale porous media with $\varepsilon_{f}=0.75$, a) $\varepsilon_{p}=0.2$, b) $\varepsilon_{p}=0.4$, c) $\varepsilon_{p}=0.6$, d) $\varepsilon_{p}=0.8$

ues obtained from the pore level simulation. A linear variation with the slope of $45^{\circ}$ between $K_{b, x x} / D^{2}$ and $\varepsilon_{f}^{3} /\left(1-\varepsilon_{f}\right)^{2}$ should exist. As seen from the figure, the computed pore level permeability values with the suggested correlation have good agreement. The suggested relation for determination of Kozeny constant yields reasonable results for the calculation of permeability for ranges of interparticle porosity between 0.4 and 0.75 when the intraparticle porosity changes from 0.2 to 0.8 .

For Kozeny constant in y direction, as can be seen from Figure 15, the change of dimensionless bulk permeability with $\varepsilon_{f}^{3} /\left(1-\varepsilon_{f}\right)^{2}$ is almost linear and is not influenced from intraparticle porosity. That's why, the Kozeny constant is found as fixed value of 125 .

Figure 17 shows the comparison of the Kozeny relation (by using $\kappa=125$ ) and obtained numerical values.

Table 2. Empirical coefficients for determination of Kozeny constant $\left(0.4<\varepsilon_{f}<0.75\right.$ and $\left.0.2<\varepsilon_{p}<0.8\right)$

\begin{tabular}{|l|l|l|l|}
\hline $\mathrm{C}_{0}$ & 4.246 & $\mathrm{D}_{0}$ & -0.112 \\
\hline $\mathrm{C}_{1}$ & 3.686 & $\mathrm{D}_{1}$ & -0.216 \\
\hline $\mathrm{C}_{2}$ & 3.115 & $\mathrm{D}_{2}$ & -0.393 \\
\hline $\mathrm{C}_{3}$ & 3.106 & $\mathrm{D}_{3}$ & -0.545 \\
\hline
\end{tabular}

As can be seen, the suggested Kozeny constant value is appropriate to provide value for permeability of the studied dual scale in y direction.

\section{CONCLUSION}

Fluid flow in a dual scale two dimensional porous media consisting of square rods are investigated, numerically. The continuity and Navier-stokes equations both for intra and interparticle pores and entire REV of dual scale porous media are solved. The intrinsic permeability values and a correlation for determination of bulk permeability tensor is suggested. Based on the obtained results following remarks can be concluded:

- for flow in intraparticle pore direction (x direction), the increase of the intraparticle pore size removes gap between the particles and provides a fully straight flow. However, for flow in transverse direction (y direction), the increase of intraparticle pore size does not have important effect on flow patterns.

- the direction of intraparticle pores has important influence on the bulk permeability. Hence, correlations for determination of bulk permeability in terms of intra- 

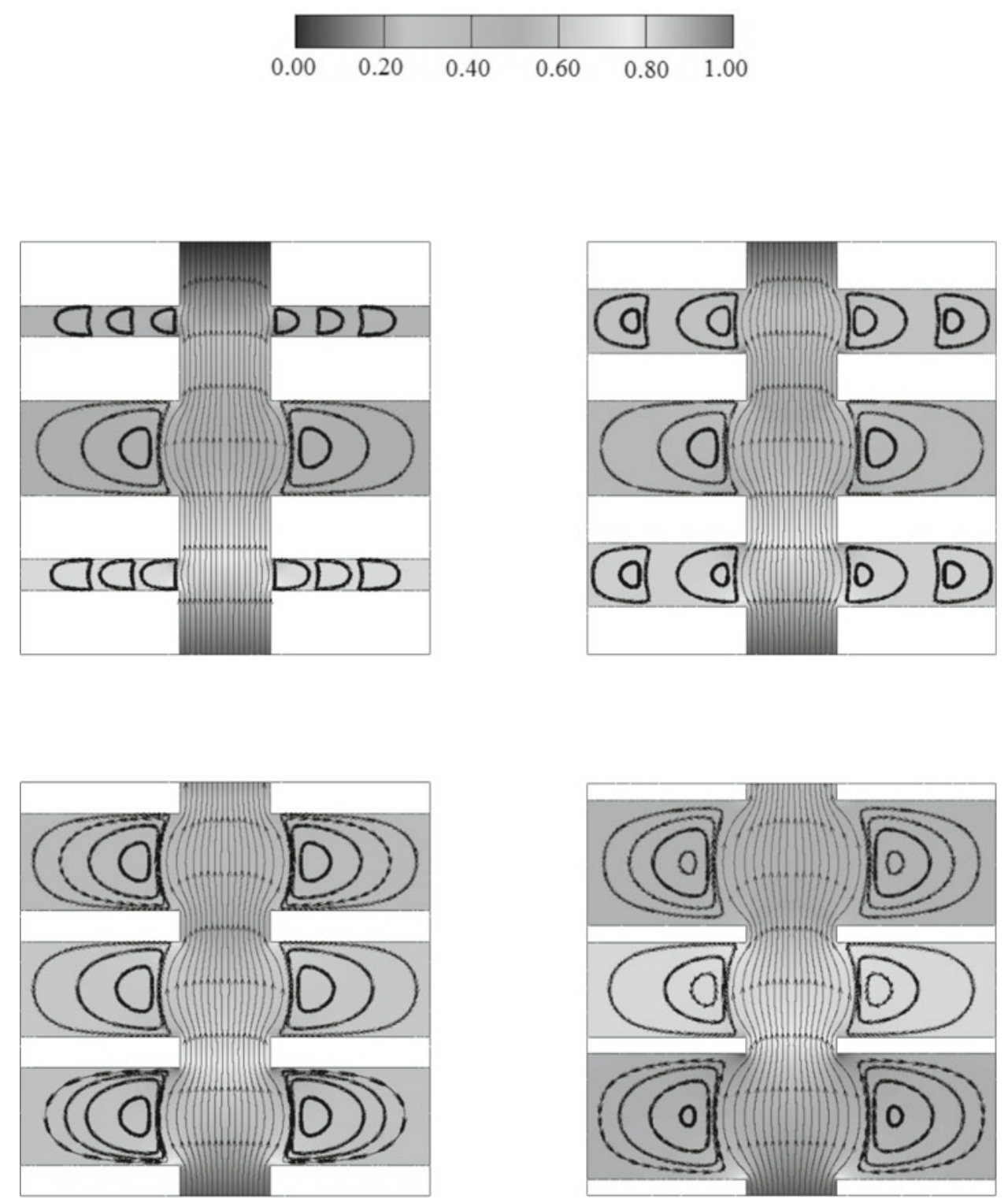

Figure 14. The streamlines and pressure contours for flow in y direction in dual scale porous media with $\varepsilon_{f}=0.4$, a) $\varepsilon_{p}=0.2$, b) $\varepsilon_{p}=$ 0.4 , c) $\varepsilon_{p}=0.6$, d) $\varepsilon_{p}=0.8$

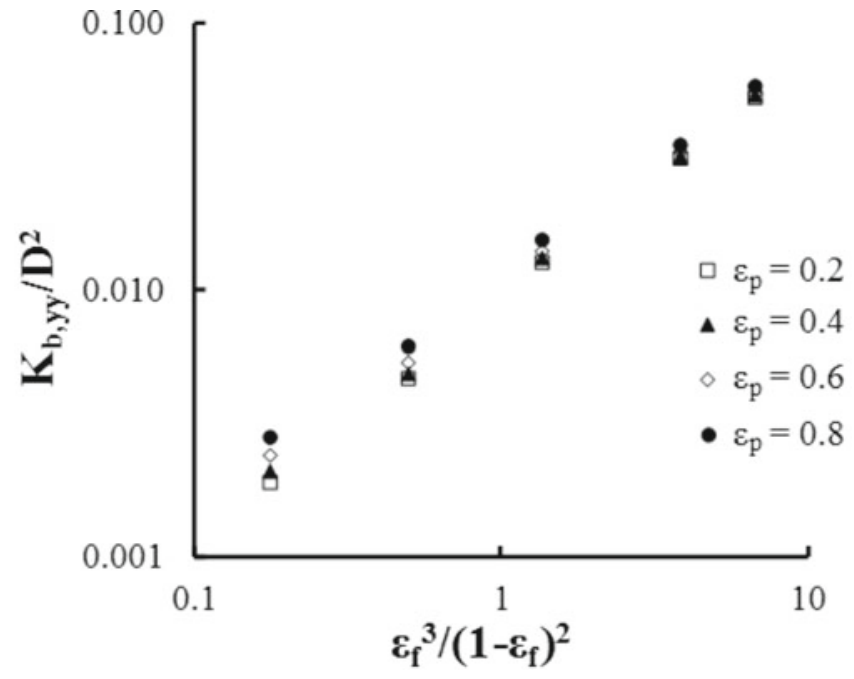

Figure 15. The change of with $K_{b, y y} / D^{2}$ with $\varepsilon_{f}^{3} /\left(1-\varepsilon_{f}\right)^{2}$

particle porosity may not be accurate for heterogeneous porous media.

- the intraparticle porosity value increase the flow rate passes through the porous media and the particle becomes more permeable. However; for high interparticle

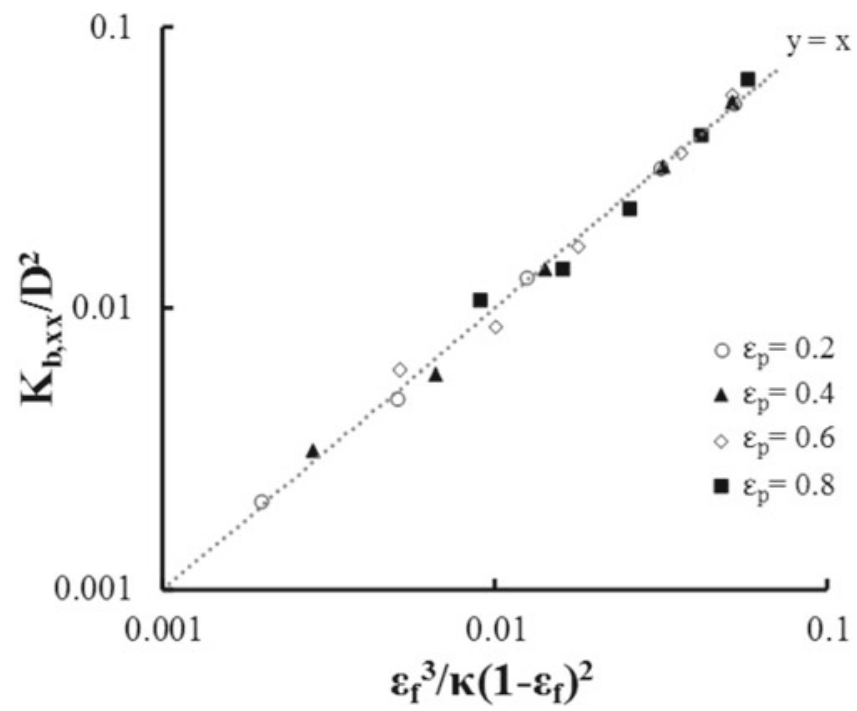

Figure 16. The comparison of suggested correlation in longitudinal direction with the obtained numerical permeability values 


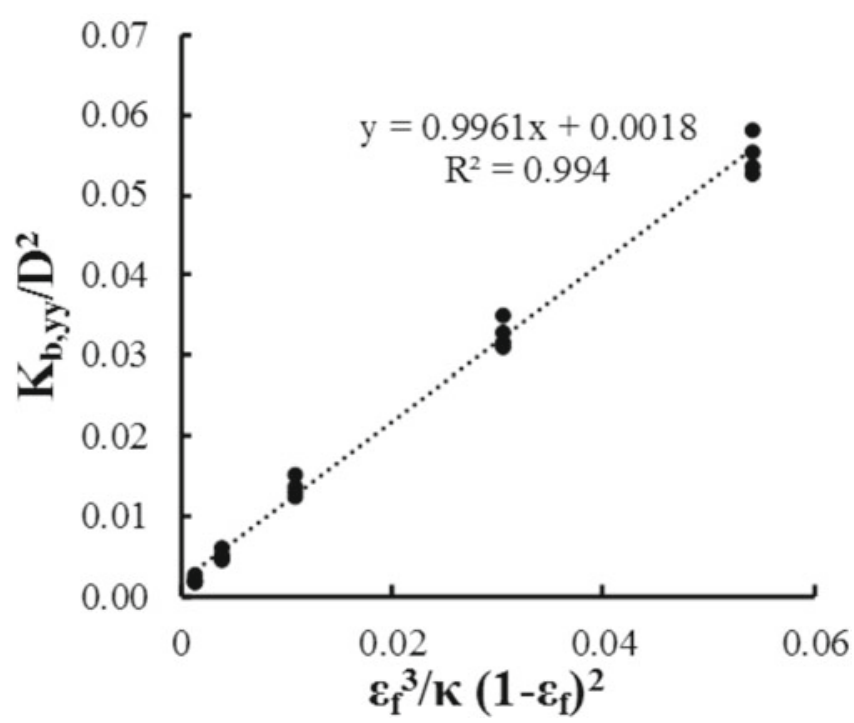

Figure 17. The comparison of the suggested equation for permeability in transverse direction with obtained numerical results

porosity values such as 0.75 , the intraparticle porosity does not have importance effect on bulk permeability.

- it seems that the equation of Kozeny-Carmen is an appropriate relation for adaption onto dual scale porous media; however the Kozeny constant should be defined in terms of intra, interparticle permeability and porosity.

\section{NOMENCLATURE}

d - Height of intraparticle pore, $m$

D - Size of square particle, $\mathrm{m}$

$\mathrm{H}-\mathrm{REV}$ dimension, $\mathrm{m}$

$\kappa \quad-$ Kozeny constant

K - Permeability, $\mathrm{m}^{2}$

L - Characteristic length, $m$

$\mathrm{p} \quad$ - Pressure, $\mathrm{Pa}$

Re - Reynolds number

$\mathrm{u}$ - Velocity in $\mathrm{x}$ direction, $\mathrm{m} / \mathrm{s}$

$\vec{u} \quad-$ Velocity vector, $\mathrm{m} / \mathrm{s}$

$u_{D} \quad-$ Darcian velocity, $\mathrm{m} / \mathrm{s}$

$\mathrm{v}$ - Velocity in $\mathrm{y}$-direction, $\mathrm{m} / \mathrm{s}$

V - Volume, $\mathrm{m}^{3}$

\section{Greek Letters}

$\varepsilon \quad-$ Porosity

$\mu \quad-$ Dynamic viscosity, $\mathrm{Ns} / \mathrm{m}^{2}$

$v \quad-$ Kinematic viscosity, $\mathrm{m}^{2} / \mathrm{s}$

@ - Density, $\mathrm{kg} / \mathrm{m}^{3}$

\section{Subscripts}

b $\quad-$ bulk

f - fluid

p - particle

$\mathrm{xx} \quad$ - longitudinal

yy - transverse

\section{LITERATURE CITED}

1. Pianko-Oprych, P. (2011). Modelling of heat transfer in a packed bed column. Pol. J. Chem. Technol. 13(4), 34-41. DOI: 10.2478/v10026-011-0046-1.

2. Tomaszewska, M. \& Bialonczyk, L. (2011). The investigation of ethanol separation by the membrane distillation process. Pol. J. Chem. Technol. 13(3), 66-69. DOI: 10.2478/ v10026-011-0040-7.

3. Nakayama, A., Kuwahara, F. \& Umemoto, T. (2002). Hayashi, T., Heat and Fluid Flow Within an Anisotropic Porous Medium. J. Heat Transf. 124(4), 746. DOI: 10.1115/1.1481355.

4. Ozgumus, T., Mobedi, M. \& Ozkol, U. (2014). Determination of Kozeny Constant Based On Porosity and Pore to Throat Size Ratio in Porous Medium with Rectangular Rods. Eng. Appl. Comp. Fluid 8, 308-318. DOI: 10.1080/19942060.2014.11015516.

5. Yu, B. \& Cheng, P. (2002). A Fractal Permeability Model for Bi-dispersed Porous Media. Int. J. Heat Mass Tran. 45, 2983-2993. DOI: 10.1016/S0017-9310(02)00014-5.

6. Papathanasiou, T.D. (2001). Flow Across Structured Fiber Bundles: A Dimensionless Correlation. Int. J. Multiphas Flow 27, 1451-1461. DOI:10.1016/S0301-9322(01)00013-1.

7. Hwang, W.R. \& Advani, S.G. (2010). Numerical Simulations of Stokes-Brinkman Equations for Permeability Prediction of Dual Scale Fibrous Porous Media. Phys Fluids 22(11), 113101. DOI: $10.1063 / 1.3484273$.

8. Ranganathan, S. (1996). A Generalized Model for the Transverse Fluid Permeability in Unidirectional Fibrous Media. Polym. Composite 17, 222-230. DOI: 10.1002/pc.10607.

9. Byon, C. \& Kim, S.J. (2013). Permeability of Mono- and Bi-dispersed Porous Media. EPJ Web of Conferences 45, 01018. DOI: $10.1051 /$ epjconf/20134501018.

10. Nield, D.A. \& Kuznetsov, A.V. (2011). Forced Convection in a Channel Partly Occupied by a Bidisperse Porous Medium: Symmetric Case. J. Heat Transf. 133(7), 072601. DOI: 10.1115/1.4003667.

11. Saada, M.A., Chikh, S. \& Campo, A. (2005). Analysis of hydrodynamic and thermal dispersion in porous media by means of a local approach. Heat Mass Transf. 42(11), 995-1006. DOI: 10.1007/s00231-005-0061-y.

12. Ngo, N.D. \& Tamma, K.K. (2001). Microscale Permeability Predictions of Porous Fibrous Media. Int. J. Heat Mass Transf. 44, 3135-3145. DOI: 10.1016/S0017-9310(00)00335-5.

13. Nedanov, P.B. \& Advani, S.G. (2002). Numerical Computation of the Fiber Preform Permeability Tensor by the Homogenization Method. Polym. Composite 23, 758-770. DOI: $10.1002 /$ pc.10474.

14. Tahir, M.W. \& Hallström, S. (2014). Åkermo, M., Effect of Dual Scale Porosity on the Overall Permeability of Fibrous Structures. Compos. Sci. Technol. 103, 56-62. DOI: 10.1016/j. compscitech.2014.08.008.

15. Wang, Q., Mazé, B., VahediTafreshi, H. \& Pourdeyhimi, B. (2006). A note on permeability simulation of multifilament woven fabrics. Chem. Eng. Sci. 61(24), 8085-8088. DOI:10.1016/j. ces.2006.09.043.

16. Nabovati, A., Llewellin, E.W. \& Sousa, A.C.M. (2010). Through-thickness permeability prediction of three-dimensional multifilament woven fabrics. Compos. Part A-Appl. S. 41(4), 453-463. DOI: 10.1016/j.compositesa.2009.11.011.

17. Tung, K.L., Shiau, J.S., Chuang, C.J., Li, Y.L. \& Lu, W.M. (2002). CFD analysis on fluid flow through multifilament woven filter cloths. Separ. Sci. Technol. 37(4), 799-821. DOI: 10.1081/SS-120002218. 